(C)2004 IEEE. Personal use of this material is permitted. However, permission to reprint/republish this material for advertising or promotional purposes or for creating new collective works for resale or redistribution to servers or lists, or to reuse any copyrighted component of this work in other works must be obtained from the IEEE. 


\title{
Individual-Cell Monitoring System in Energy Backup Banks
}

\author{
Cesar Ortega, Jaime Orozco, Jojutla Pacheco and Alejandro Rivera \\ Inst. of Electrical Research / Dept. of Control and Instrumentation \\ Cuernavaca, MEXICO \\ ortegas@iie.org.mx
}

\begin{abstract}
This paper presents the characteristics of a lowcost individual-cell monitoring system suitable for batterybased backup systems. The system is being developed at the Department of Control and Instrumentation (GCI), Institute of Electrical Research (IIE), Mexico. The system has three main components: a central unit that stores, analyses and presents cells data, a data concentrator module that gathers data and sends it to the central unit and multiple data acquisition modules, one for each monitored cell. The concentrator can also work as a low-cost charge-controller for lead-acid batteries connected to photovoltaic panels. This paper also presents results from the field tests applied to the charge controller.
\end{abstract}

\section{INTRODUCTION}

Lead-Acid batteries (LAB) are widely used in industry to store energy in chemical form and deliver it as electricity. LABs can be found in electric vehicles, photovoltaic installations, power plants and backup systems in critical installations such as hospitals, computer centers, telecommunication sites, oil-rigs and emergency bays in roads. However, their electrochemical nature gives them a very short useful life.

Much research has been conducted to understand the phenomena that take place inside a LAB during its charge and discharge cycles; phenomena that affect in the long term the battery useful life $[1,2,3,4,5]$. These studies have provided two conclusions:

a) The selection of the right charging algorithm can extend the useful life of a battery and,

b) A continuous monitoring helps to estimate the LAB's state-of-health (SOH) and hence, it is useful to locate the batteries that are about to fail. This allows maintenance personnel to take action before a contingency arises.

A LAB is a set of lead-acid cells connected in series. In this paper the acronym LAB will be used to refer to both lead-acid batteries and cells.

LABs are very delicate devices whose useful life strongly depends on factors such as operation temperature, charge and discharge regimes and connections with other elements. In energy backup systems, LABs are connected into arrays to achieve operational voltage and current levels. Connecting LABs in series elevates voltage. High current rates are achieved by connecting LABs in parallel. A combination of series and parallel arrays can deliver almost any voltage and current specification.

Reliable operation of a LAB-array depends on every element functioning correctly. In a series array, if one LAB fails, then the complete array fails causing maintenance costs and the consequent drop in system reliability. The useful life of a particular $\mathrm{LAB}$ in an array depends on several factors such as:

- Difference in the ability to accept charge with respect to other supposedly identical cells.

- Excessive electrolyte loss.

- Thermal stress if the cell is located at the end of the array.

Individual and continuous monitoring of LABs can detect elements with higher fail-probability, allowing their replacement before system breakdown, hence increasing the reliability of the backup system.

In recent years, monitoring LAB-banks has become a critical activity in companies that require an uninterruptible power supply. Some benefits of monitoring the LAB-banks are:

- Increase in system reliability.

- Reduction in maintenance costs.

- Extension of LAB's useful life.

- Personnel safety.

The parameters that are commonly monitored to determine the LAB's SOH are:

- LAB-bank voltage.

- Individual LAB voltage.

- Temperature (environment and at the LAB)

- LAB's internal resistance.

- Resistance between LABs.

- Number of charge-cycles.

Typically, these parameters are registered from 1 to 24 times a day; but depending on the application, longer sample rates could be recommended.

This paper presents the characteristics of a low-cost monitoring system suitable for LAB-based backup systems. The system is being developed at the Department of Control and Instrumentation (GCI), Institute of Electrical Research (IIE), Mexico. 


\section{SYSTEM DESCRIPTION}

The system has three main components: a central station that stores, analyses and presents data; one or several data concentrator modules and multiple data acquisition modules, one for each monitored LAB. Figure 1 shows a diagram of the system.

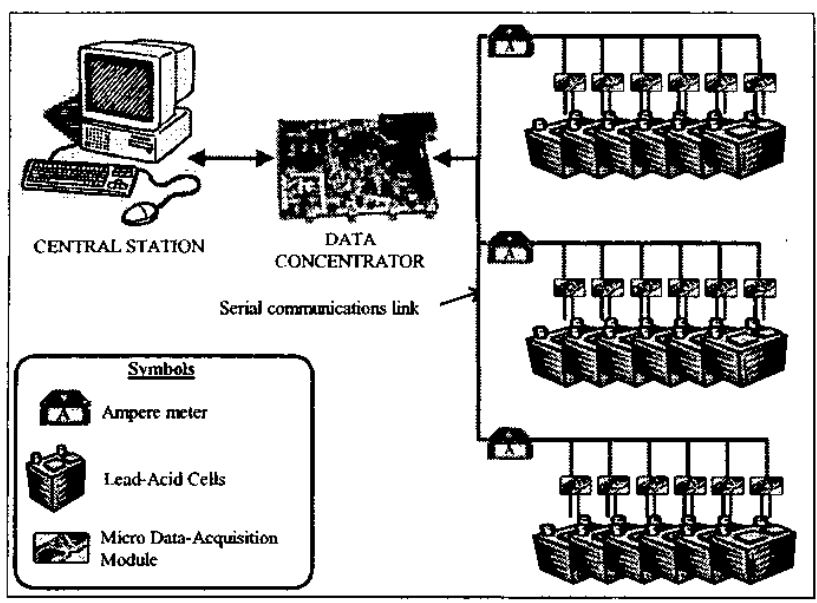

Fig. 1. Diagram of the cell-monitoring system

\section{A. Data Acquisition Modules}

Each data-acquisition module registers voltage and temperature of the corresponding cell. These modules have a low-cost, low-power, commercial microcontroller each. The microcontroller performs the data acquisition and the communications with data concentrators. Figure 2 shows a photograph of a data acquisition module without its case.

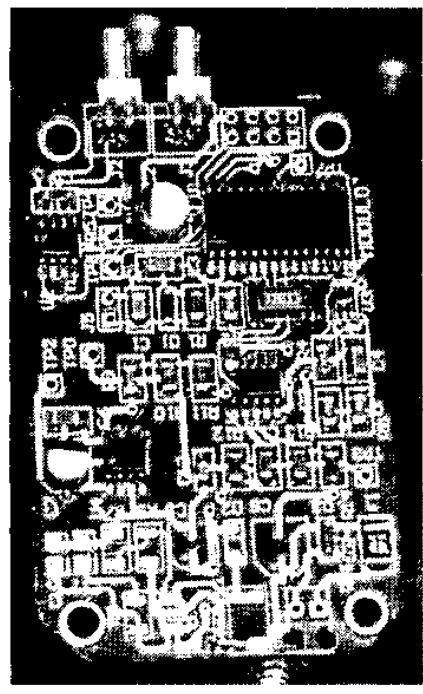

Fig. 2. Photograph of a data acquisition module.

Every module takes its power from the $\mathrm{LAB}$ it is monitoring. An RS-485 network is used to communicate all the acquisition modules in a bank with the corresponding data concentrator. In order to avoid the problems associated with differential power supplies, every acquisition module has an isolated power supply for its communications circuitry. Communications are carriedout using a proprietary protocol that reduces overhead in order to save the high-energy demands that occur during transmissions.

In its latest version, the system can store data from up to 2,048 LABs. Data are stored in a commercial database located at the central station.

\section{B. Data Concentrators}

A system may have one or several data concentrators, depending on its size. There must be one concentrator for every array of LABs connected in series. The concentrator is also a microcontroller-based board. The same microcontroller that is used in de acquisition modules is used in the concentrator.

Figure 3 shows a photograph of one concentrator without its case.

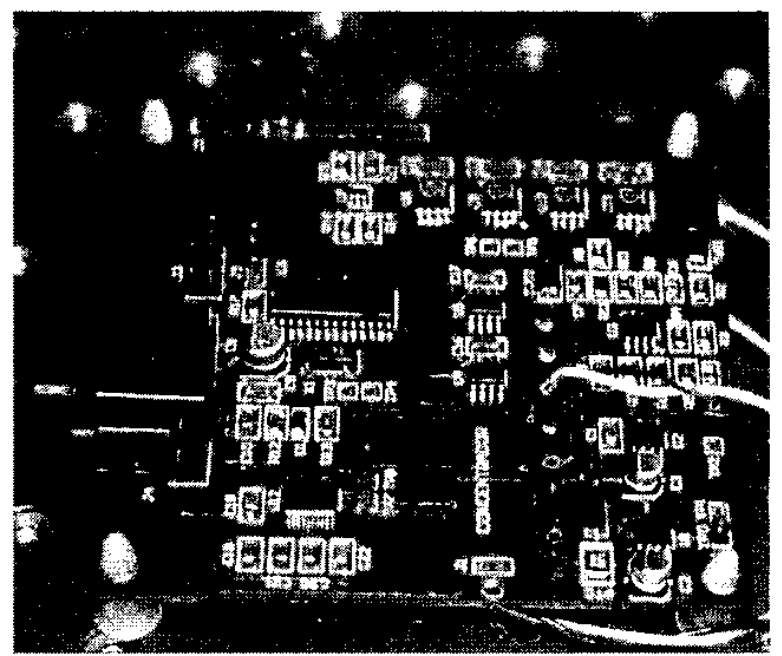

Fig. 3. Photograph of a data concentrator.

Every data concentrator in the system will perform the following functions:

- Periodic data collection from its associated acquisition modules.

- Monitoring of bank's current.

- Monitoring of room's temperature.

- Communication with the central station via a serial channel that can be, for example, a low-power radio or an RS-485 network.

Data are stored in the concentrator until the central station requests them. This scheme assumes that the concentrators are located in the same room as the LABs being monitored, and that the central station is located in a different room.

The user sets the sampling rate at which the central station reads data from the concentrators. This parameter can take values between 1 hour and a month. Faster 
sampling rates require the use of larger databases. For small, non-critical applications, slow sampling rates are recommended.

\section{Central Station}

The central station must be a computer with enough processing power and disk space to store, analyze and display all data collected by the concentrators. The number of monitored LABs vary from application to application. Any number between some tens to some hundreds is possible. The central station performs the following functions:

- Serial communication with the concentrators using a proprietary protocol.

- Database management and updating.

- Monitoring-system administration.

- Estimates SOH of each LAB in the system.

- Graphic display of collected data.

- Report generation.

The programs of the central station are written in LavView $\left.{ }^{(}\right)$by National Instruments ${ }^{\mathrm{TM}}$. This programming tool permits adapting the program to any particular user need. It is also possible to make the LAB-bank's data available over the Internet, so that remote supervision can be achieved.

\section{DATA Visualization}

The central station presents data in graphic form. Two types of graphs are possible: Bar-graphs where comparisons between LABs in the same bank can be done; and time-graphs where the behavior of the LABs over a period of time can be observed. Bar-graphs can help system operators to detect a particular LAB whose voltage or temperature is prominently lower or higher than the rest of the LABs in the same bank. Time-graphs can help to detect abnormal trends in voltage or temperature over time. Figures 4 and 5 show examples of each type of graph. Data correspond to a real 8-LAB bank installed in our laboratory for system testing purposes. Notice that one of the LABs is faulty. This is prominently displayed in both graphs. The information provided by these graphs can help an experienced system operator to detect those LABs more prone to fail and take the corresponding preventing maintenance actions.

The bar-graphs window is divided into a bottom and a top graph. The bottom one presents data of 50 cells. Data consist of the voltage and temperature registered in each $\mathrm{LAB}$ at a particular date and time. The maximum, minimum and average values for each variable are indicated on the same bar. A slider scrolls through all the LABs in a bank, so it is always possible to compare the parameters of $50 \mathrm{LABs}$ at-a-glance. To have a better look of details, the top graph shows data of only 10 LABS. The LABs presented on both graphs are selected by the same slide-control.
The slider at the bottom of the graphs selects the date and time for which data will be displayed. A continuous movement of this control, creates a sort of animation in the bar-graphs. This visual effect helps detecting cells that presented big variations in their parameters at a particular point in time.

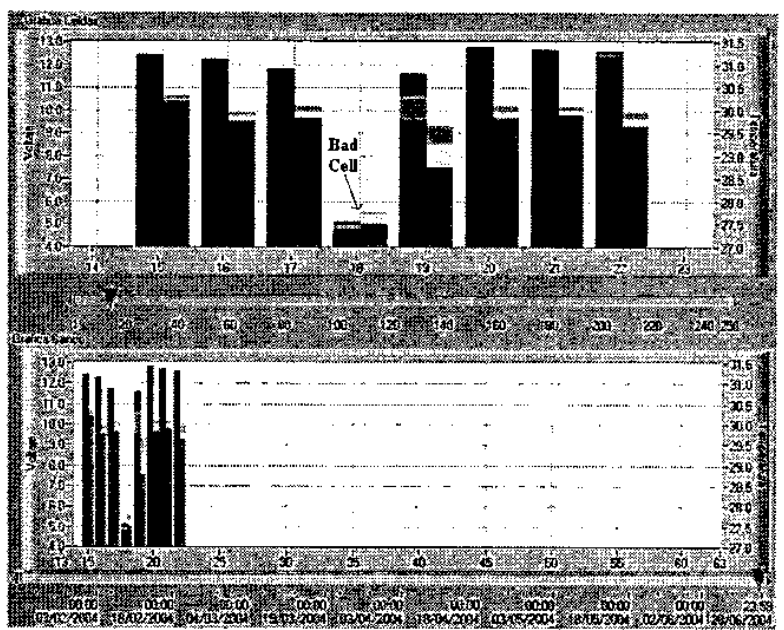

Fig. 4. Bar-graph of voltage and temperature in an 8-LAB bank

Time-graphs show the behavior in time of the parameters of a set of LABs. LABs to be displayed are selected by highlighting their number in a selector located at the bottom of the graph. When looking for abnormal behaviors, it is desirable to display data from many cells at the same time. By doing so, abnormal behavior appears as spikes. These can be easily located by adjusting dates and scales. Figure 5 shows the case when one LAB is presenting a voltage much lower than the others. Even its temperature seems to be a couple of degrees below the average for the bank. But this can be due to a bad calibration of the temperature sensor.

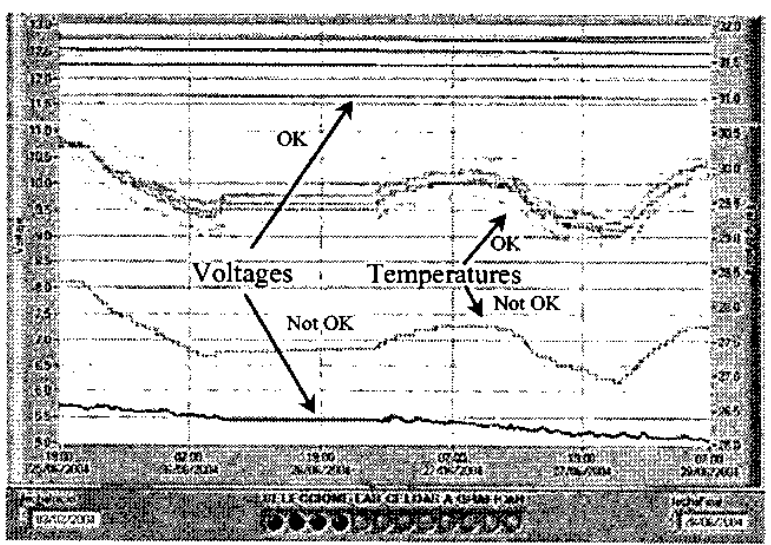

Fig. 5. Time-graphs of voltage and temperature in an 8-LAB bank 
When looking for small differences between LABs, it is recommended to display only two or three graphs at the same time. Figure 6 shows a detail of the graphs shown in figure 5. Data corresponding to only the abnormal LAB and another one are presented. Notice the similarity between the two temperature graphs. Although there is a difference of a couple of degrees, it is obvious that none of the LABs has suffered thermal stress during the time of monitoring. Exact measurements are not necessary, as long as the monitoring in one particular LAB is consistent.

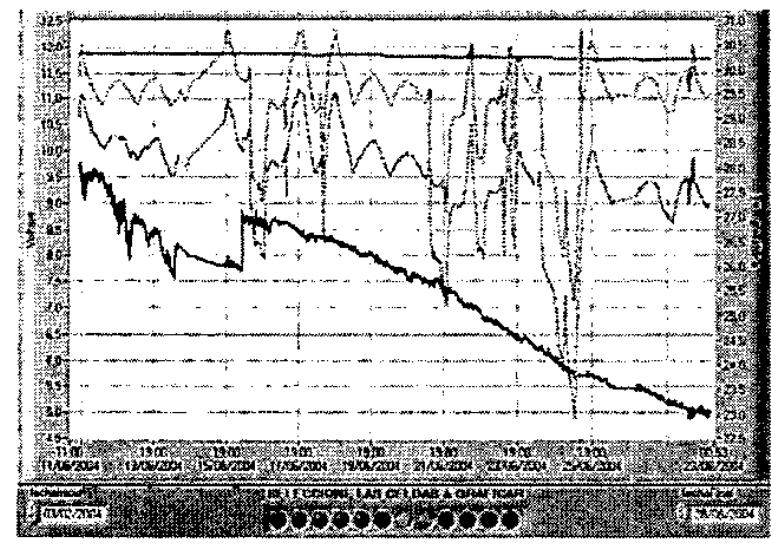

Fig. 6. Time-graphs of two LABs. One of them in fail.

In figure 6, the two bottom graphs correspond to a faulty LAB with a voltage out of specification. The top graphs show voltage and temperature of a healthy LAB. In energy backup systems, LAB's voltages should vary very little and temperature should be strongly correlated to that of the environment. When energy is demanded, both voltages and temperatures should present similar variations. If one LAB behaves differently, it should be marked as suspicious and the appropriate diagnosis procedure applied.

The voltage and temperature sensors used have a tolerance of $\pm 1 \%$. Hence, differences between the values read in different LABs seem significant. However, the purpose of the system is to detect abnormal changes in LAB's behavior, therefore, the exactness of a sample is not so relevant as it is its consistency.

\section{CONCLUSIONS AND FUTURE WORK}

A monitoring system for large battery-banks has been presented. All components (hardware and software), were developed at the Institute of Electrical Research in Mexico. The system provides the following benefits:

- Opportune detection of failing LABs. With this, preventive maintenance actions can be scheduled, which is cheaper than corrective maintenance during emergencies.

- Longer useful life of LABs. By observing the trend in the voltage of a $\mathrm{LAB}$, it is possible to determine if some maintenance action is needed, for example, replenish electrolyte or check the connections. Opportune maintenance actions can make the LAB's useful life longer.

- Low cost.

- Adaptability. Since all hardware modules are programmable, it is possible to adapt the system to particular user requirements.

Since the IIE mission is to develop technology and not to commercialize it, it is intended that the system will be transferred to a private company in the future. Before that, more laboratory and field tests should be performed.

\section{REFERENCES}

[1] Catherino H., Burgel J., Rusek A. and Feres F., "Modeling and simulation of lead-acid battery charging", J. of Power Sources, Vol.80, 1999, pp.17-20

[2] Copetti J. and Chenlo F., "Lead/acid batteries for photovoltaic applications. Test results and modeling", J. of Power Sources, Vol.47, 1994, pp.109-1 18

[3] Geuer W., "Investigations of the Aging Process in Lead-Acid-Batteries", Tesis, RWTH Aachen, 08.05.1992

[4] Gross S: Analytical modelling of battery cycle life. J Power Sources 12: 317-322, 1984

[5] Salkind A., Atwater T., Singh P., Nelatury S., Damodar S., Fennie C., Reisner D., "Dynamic characterization of small lead-acid cells", J. of Power Sources, Vol.96, 2001, pp. 151-159 